ఠ

\title{
miR-195 enhances the radiosensitivity of colorectal cancer cells by suppressing CARMI
}

This article was published in the following Dove Press journal:

OncoTargets and Therapy

20 February 2017

Number of times this article has been viewed

\author{
Li Zheng* \\ Jiangtao Chen* \\ Zhongyong Zhou \\ Zhikuan He \\ Department of General Surgery, \\ Huaihe Hospital of Henan University, \\ Kaifeng, People's Republic of China \\ *These authors contributed equally \\ to this work
}

Background: microRNAs (miRNAs) can regulate the sensitivity of cancer cells to chemotherapy and radiotherapy. Aberrant expression of miR-195 has been found to be involved in colorectal cancer (CRC); however, its function and underlying mechanism in the radioresistance of CRC remains unclear.

Methods: The levels of miR-195 and CARM1 were detected by quantitative reverse transcriptionpolymerase chain reaction and Western blot analysis in HCT-116 and HT-29 cells, respectively. Colony survival and apoptosis were determined by clonogenic assay and flow cytometry analysis, respectively. The apoptosis-related proteins $\mathrm{Bax}, \mathrm{Bcl}-2$, and $\gamma$-H2AX were detected using Western blot. The targets of miR-195 were identified by bioinformatic prediction and luciferase reporter assays. CRC cells in vitro and in vivo were exposed to different doses of X-ray radiations.

Results: miR-195 was downregulated, and CARM1 was upregulated in HCT-116 and HT-29 cells. miR-195 overexpression or CARM1 knockdown suppressed colony survival, induced apoptosis, promoted expression of Bax and $\gamma$-H2AX, and inhibited Bcl-2 expression in CRC cells. CARM1 was identified and validated to be a functional target of miR-195. Moreover, restored expression of CARM1 reversed the enhanced radiosensitivity of CRC cells induced by miR-195. Furthermore, miR-195 increased the sensitivity of CRC cells to radiation in vivo.

Conclusion: miR-195 enhances radiosensitivity of CRC cells through suppressing CARM1. Therefore, miR-195 acts as a potential regulator of radioresistance for CRC cells and as a promising therapeutic target for CRC patients.

Keywords: miR-195, CARM1, colorectal cancer, radiosensitivity

\section{Introduction}

Colorectal cancer (CRC) is the most common malignant tumor, and it is one of the major causes of cancer deaths. ${ }^{1}$ The total rate of CRC risk is generally about $5 \%$, and the 5-year survival rate remains at around $50 \%{ }^{2}$ To date, surgery is a mainstay of treatment for $\mathrm{CRC}$; however, surgery alone is insufficient, due to the fact that CRC is often diagnosed at an advanced stage. ${ }^{3}$ Although preoperative treatment with chemoradiotherapy in combination with conventional surgery was developed, it failed to universally improve outcomes as a result of chemoresistance and radioresistance. Therefore, there is urgent need to improve radiosensitivity in patients with CRC and understand the mechanisms involved in radiation resistance of CRC.

Coactivator-associated arginine methyltransferase 1 (CARM1), a protein with arginine-specific histone methyltransferase activity, was initially identified as an SRC-2 binding protein in a yeast two-hybrid screening, and it functioned as a coactivator for many nuclear receptors and transcription factors. ${ }^{4,5}$ A previous study reported that CARM1 could enhance breast cancer cell migration and metastasis by promoting the methylation of BAF155. ${ }^{6}$ Moreover, another report found that CARM1 could increase
Department of General Surgery, Huaihe Hospital of Henan University, No 8 Baobei Road, Gulou District, Kaifeng 475000, People's Republic of China

Tel $+86037 \mid 23906900$

Email lizhengccn@।63.com
OncoTargets and Therapy 2017:10 1027-1038

1027

Dovepress in

http://dx.doi.org/|0.2147/0TTS125067 (c) (1) (5) 2017 Zheng et al. This work is published and licensed by Dove Medical Press limited. The full terms of this license are available at https://www.dovepress.com/terms.php cc. hereby accept the Terms. Non-commercial uses of the work are permitted without any further permission from Dove Medical Press Limited, provided the work is properly attributed. For permission for commercial use of this work, please see paragraphs 4.2 and 5 of our Terms (https://www.dovepress.com/terms.php). 
the chemosensitivity of breast cancer cells by methylation of MED $12 .{ }^{7}$ However, the function of CARM1 on radioresistance was unclear.

microRNAs (miRNAs), a class of small noncoding RNA containing about 18-24 nucleotides, function as posttranscriptional regulators and usually lead to translational suppression or RNA degradation via specific binding to $3^{\prime}$-untranslated region ( $3^{\prime}$-UTR) of their target genes. ${ }^{8,9}$ miRNAs could serve as oncogenes or tumor suppressor genes involved in nearly all cancers. ${ }^{10}$ Recent studies have reported that some miRNAs were involved in regulating radiation response of different cancers to increase susceptibility or protect cells from radiation effect. ${ }^{11-15}$ For instance, Lin et $\mathrm{al}^{15}$ found that the miR-200c expression was downregulated in breast cancer cells, and miR-200c overexpression could increase the radiosensitivity of breast cancer cells by inhibiting cell proliferation, and increasing apoptosis and DNA double-strand breaks through directly targeting TANK-binding kinase 1 .

miR-195 expression varies from one cancer to another, and its functional effect is also diverse in different malignancies. ${ }^{16,17}$ In addition, aberrant expression of miR-195 was found in CRC cells. For example, miR-195 was downregulated in CRC cells, and restoration of miR-195 reduced cell viability, promoted cell apoptosis, and suppressed tumorigenicity by directly targeting Bcl-2. ${ }^{18}$ Moreover, Zhu et $\mathrm{al}^{19}$ illustrated that overexpression of miR-195 increased the radiosensitivity of breast cancer cells through inhibiting Bcl-2. However, the role and molecular mechanism of miR-195 in CRC radioresistance is still unclear.

In the present study, we aimed to investigate the effect of miR-195 on the radiosensitivity of CRC cells and its underlying molecular mechanisms.

\section{Materials and methods Cell culture and transfection}

Normal human colonic epithelial cell line (HCEpiC) and human CRC cell lines (HCT-116 and HT-29) were purchased from the American Type Culture Collection (ATCC, Manassas, VA, USA) and cultured at $37^{\circ} \mathrm{C}$ in humidified $5 \%$ $\mathrm{CO}_{2}$. All cells were grown in RPMI 1640 (Gibco, Grand Island, NY, USA) supplemented with 10\% fetal bovine serum (FBS; Gibco), 100 IU/mL penicillin (Invitrogen, Carlsbad, $\mathrm{CA}, \mathrm{USA}$ ), and $100 \mu \mathrm{g} / \mathrm{mL}$ streptomycin (Invitrogen). miR-control, miR-195 mimics, and miR-195 inhibitor (anti-miR-195) were purchased from Dharmacon (Lafayette, CO, USA). Empty pcDNA vector (pcDNA-control), small interfering RNA against CARM1 (si-CARM1), and its negative control (si-control) were obtained from Dharmacon.
Cells $\left(1 \times 10^{4} /\right.$ well) were inoculated in a 96 -well plate and cultured for $24 \mathrm{~h}$ so that they could attach to the bottom of the well, and then transfection was performed using Lipofectamine 2000 (Invitrogen) in accordance with the protocol provided by the manufacturer. Cells were used for further analysis $48 \mathrm{~h}$ post transfections.

\section{Quantitative reverse transcription- polymerase chain reaction ( $\mathrm{qRT}-\mathrm{PCR}$ )}

Total RNA was extracted from HCEpiC, HCT-116, and HT-29 cells with TRIzol Reagent (Invitrogen). M-MLV reverse transcriptase (Invitrogen) was used to synthesize specific cDNA according to the instruction provided by the manufacturer. The expression of miR-195 and CARM1 mRNA was quantified using the SYBR Green qRT-PCR master mix on ABI PRISM 7900HT Sequence Detection System (Applied Biosystems, Foster City, CA, USA). U6 snRNA and $\beta$-actin served as endogenous controls. The primer sequences were as follows: for miR-195 5'-CGTAGCAGCACAGAAAT-3' (forward) and 5'-GTGCAGGGTCCGAGGT-3' (reverse); for CARM1 5'-TTGATGTTGGCTGTGGCTCTGG-3' (forward) and 5'-ATGGGCTCCGAGATGATGATGTCC-3' (reverse); for U6 5'-CTCGCTTCGGCAGCACA-3' (forward) and 5'-AACGCTTCACGAATTTGCGT-3' (reverse); for $\beta$-actin $5^{\prime}$-GCGAGAAGATGACCCAGATC-3' (forward) and 5'-CGGTTGGCCTTAGGGTTCAGGGGGG-3' (reverse). The relative expression of mRNAs was calculated and normalized to the control gene using the $2^{-\Delta \Delta \mathrm{Ct}}$ method.

\section{Western blot analysis}

The primary antibody, anti-CAMR1 (1:1,000; sc5418), was purchased from Upstate Biotechnology (Lake Placid, NY, USA). Anti-Bax (1:1,000; clone 6A7), anti-Bcl-2 (1:1,000; Cat sc-509), and anti- $\gamma-H 2 A X(1: 1,000$; Ser139) were acquired from Santa Cruz Biotechnology (Santa Cruz, CA, USA). Anti$\beta$-actin (1:10,000; A1978) was obtained from Sigma-Aldrich (Louis, MO, USA). All these antibodies are monoclonal antibodies. Cells were harvested and total proteins were isolated with RIPA reagents (Thermo Scientific, Rockford, IL, USA), according to the manufacturer's instruction. Subsequently, protein concentrations were detected using the BCA kit (Pierce, Rockford, IL, USA). Western blot analysis was performed following previous protocols. ${ }^{20}$ Briefly, protein specimens were isolated by a $10 \%$ sodium dodecyl sulfatepolyacrylamide gel electrophoresis (SDS-PAGE) and then transferred to polyvinylidene fluoride membranes (Millipore, Billerica, MA, USA). After blocking in Tris Buffered saline with Tween (TBST) buffer with $5 \% \mathrm{BSA}$ for $2 \mathrm{~h}$ at $37^{\circ} \mathrm{C}$, the 
membranes were incubated with primary antibodies overnight at $4^{\circ} \mathrm{C}$. Following washing in TBST, the membranes were further incubated with secondary antibody labeled with horseradish peroxidase for $1 \mathrm{~h}$ at room temperature. The proteins were visualized using the Image Quant software (Molecular Dynamics, Sunnyvale, CA, USA).

\section{Clonogenic assay}

Cells $\left(2 \times 10^{5} /\right.$ well $)$ were inoculated in 6-well plates, and transfection was carried out at $24 \mathrm{~h}$. Forty-eight hours post transfection, cells were seeded into $100-\mathrm{cm}$ dishes and treated with variable doses $(0,2,4,6$, and 8 Gy) of X-rays. After incubation for 14 days, colonies formed were stained with $1 \%$ crystal violet, and colonies consisting of more than 50 cells were counted. Plate efficiency was calculated using the following formula: (number of colonies/number of cells inoculated) $\times 100 \%$. Survival fraction was normalized to the plating efficiency of control groups. The data were fitted to linear-quadratic model using Sigmaplot software (Systat, San Jose, CA, USA).

\section{Flow cytometry}

Transfected cells were irradiated with a dose of 4 Gy and cultured for $24 \mathrm{~h}$. Then the cells were harvested by trypsinization, washed twice with cold phosphate-buffered saline, and suspended in $500 \mathrm{~mL}$ of binding buffer and stained using $5 \mu \mathrm{L}$ Annexin V/FITC and $5 \mu \mathrm{L}$ PI (Sigma, CA, USA) for $15 \mathrm{~min}$ at room temperature in the dark. Stained cells were identified and analyzed using a FACScan flow cytometer (Becton Dickinson, CA, USA). The flow cytometry data were analyzed using BD FACS Diva software V6.1.3 (BD Biosciences, San Jose, CA, USA).

\section{Luciferase reporter assays}

The wild CARM1 3'-UTR and mutated CARM1 3'-UTR (a site-directed mutagenesis of miR-195 binding site introduced in CARM1 3'-UTR) were amplified and cloned into downstream of the luciferase gene in a pGL3 control plasmid. The constructed vectors were named as WT-CARM1-PGL3 and MUT-CARM1-PGL3, respectively. HCT-116 and HT-29 cells co-transfected with WT(MUT)-CARM-PGL3 and miR-control or miR-195 mimics and cultured for $48 \mathrm{~h}$. Then, luciferase activities were measured using the Dual-Luciferase Reporter Assay System (Promega, Madison, WI, USA), according to the protocol provided by the manufacturer.

\section{Tumor formation in nude mice}

A total of $5 \times 10^{6}$ HT-29 cells transfected with miR-195 or miR-control were subcutaneously injected into 4- to 6-week-old female BALB/c nude mice ( $\mathrm{n}=6$ per group). The mice were exposed to a dose of 8 Gy X-rays when the tumor volume was about $100 \mathrm{~mm}^{3}$. The tumor volumes were measured with calipers every 5 days after radiation according to the formula: volume $=0.5 \times$ length $\times$ width $^{2}$. At Day 20 after radiation, mice were sacrificed and imaged, and tumor weight was detected. All animal procedures were performed with the approval of the Local Medical Experimental Animal Care Commission. All animal-handling procedures were performed according to the Guide for the Care and Use of Laboratory Animals of the National Institutes of Health and followed the guidelines of the Animal Welfare Act.

\section{Statistical analysis}

All analyses were performed using SPSS 19.0 software (IBM, Armonk, NY, USA). The data were expressed as mean \pm SD. The Student's $t$-test or one-way ANOVA was used to evaluate the statistical significance. A $P$-value of less than 0.05 was considered statistically significant.

\section{Results}

\section{miR-I 95 is downregulated and CARMI is upregulated in CRC cell lines}

The level of expression of miR-195 and CARM1 in human corneal epithelial cells (HCEpiC) and CRC cell lines (HCT116 and HT-29) was measured using qRT-PCR and Western blot. miR-195 expression was significantly lower in HCT-116 and HT-29 cells compared with HCEpiC cells (Figure 1A). CARM1 protein levels were significantly higher in HCT-116 and HT-29 cells compared with HCEpiC cells (Figure 1B). These results suggested that miR-195 and CARM1 may be involved in the pathogenesis of CRC.

\section{miR-I95 enhances radiosensitivity of CRC cells}

The colony survival assay is regarded as a typical standard to determine radiosensitivity. ${ }^{21}$ Moreover, the anti-apoptosis ability of cancer cells is closely related to radioresistance. To examine whether miR-195 affected radiosensitivity of CRC cells, HCT-116 and HT-29 cells transfected with miR-195 mimics or miR-control were exposed to different doses of ionizing radiation $(0,2,4,6$, and $8 \mathrm{~Gy})$. The results of clonogenic assay indicated that overexpression of miR-195 significantly reduced the survival fractions of cellular colony at each dose (2, 4, 6, and 8 Gy) in HCT-116 (Figure 2A) and HT-29 cells (Figure 2B) compared with controls. Furthermore, cells transfected with miR-195 mimics or miR-control were treated with a dose of $4 \mathrm{~Gy}$ 

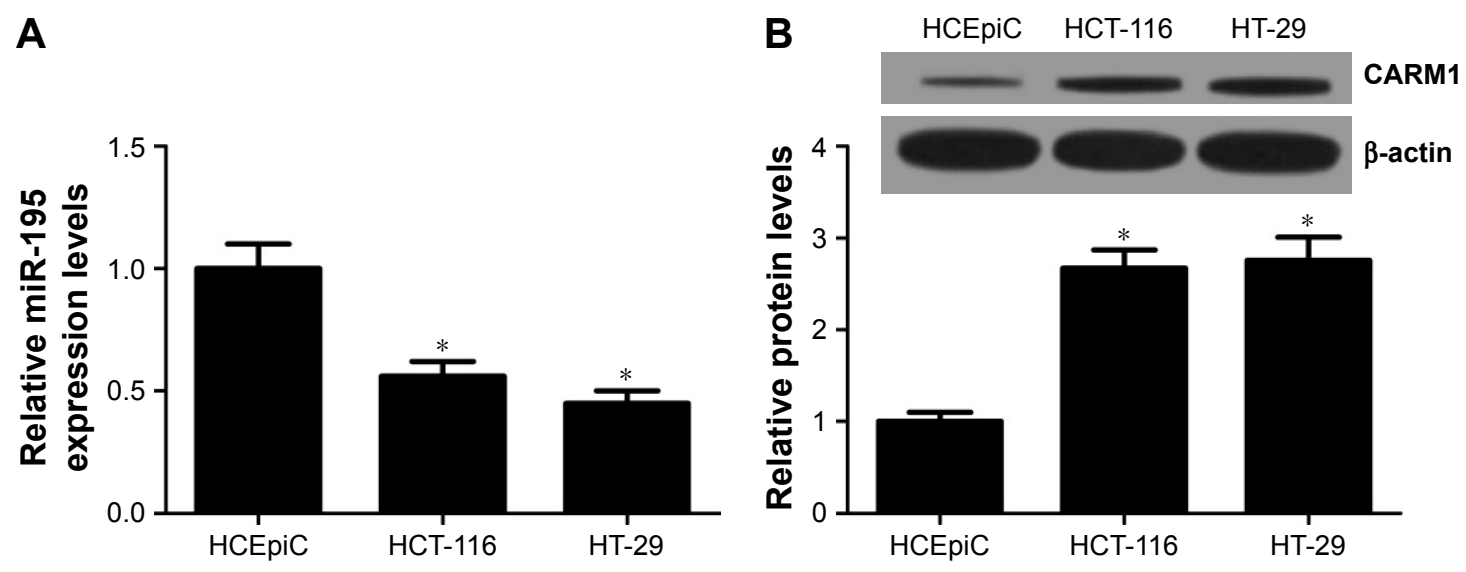

Figure I The expression of miR-195 and CARMI in CRC cell lines HCT-II6 and HT-29.

Notes: (A) qRT-PCR analysis of the expression levels of miR-195 in HCT-I I6, HT-29 cells, and HCEpiC cells. (B) The protein level of CARMI was detected and quantified in HCT-I 16, HT-29, and HCEpiC cells by Western blot. $* P<0.05$ vs control group.

Abbreviations: qRT-PCR, quantitative reverse transcription-polymerase chain reaction; CRC, colorectal cancer.

ionizing radiation. Flow cytometry analysis showed that miR-195 overexpression strikingly increased the apoptosis rates of HCT-116 (Figure 2C and D) and HT-29 cells (Figure 2E and F), and the effect was more pronounced in the presence of ionizing radiation. Western blot was performed to detect the expression of apoptosis-related proteins Bax, $\mathrm{Bcl}-2$, and $\gamma$-H2AX. The results demonstrated that miR-195 overexpression increased the levels of Bax and $\gamma-\mathrm{H} 2 \mathrm{AX}$, and reduced Bcl-2 expression in HCT-116 (Figure 2G) and HT-29 cells (Figure 2H), and the effect was much stronger when combined with ionizing radiation. Taken together, these results revealed that miR-195 enhanced sensitivity of $\mathrm{CRC}$ cells to radiation treatment.

\section{CARMI knockdown enhances radiosensitivity of CRC cells}

To confirm the effect of CARM1 on radiosensitivity of CRC cells, HCT-116 and HT-29 cells transfected with si-control or si-CARM1 were treated with various doses of ionizing radiation $(0,2,4,6$, and $8 \mathrm{~Gy})$, respectively. The clonogenic assay indicated that the CARM1 knockdown by si-CARM1 significantly reduced the survival fractions of cellular colony at each dose (2, 4, 6, and 8 Gy) in HCT-116 (Figure 3A) and HT-29 cells (Figure 3B) compared with controls. Western blot analysis showed that radiation treatment could lower the level of CARM1 in HCT-116 and HT-29 cells (Figure 3C and D). Moreover, flow cytometry analysis showed that a combination of si-CARM1 and radiation treatment resulted in a higher apoptosis rate than a single treatment (si-CARM1 or radiation) in HCT-116 (Figure 3E) and HT-29 cells (Figure 3F). Furthermore, CARM1 knockdown by si-CARM1 upregulated the level of Bax and $\gamma$-H2AX expression, and downregulated Bcl-2 expression in HCT-116
(Figure 3G) and HT-29 cells (Figure 3H), and the effect was much stronger when co-treated with ionizing radiation. These results suggested that CARM1 knockdown enhanced sensitivity of CRC cells to radiation treatment.

\section{CARMI is a direct target of miR-195 in CRC cells}

miRNAs exert functional effects mainly by base-pairing with the complementary sequence of their targets. An miRNA target analysis tool, TargetScan, was used to predict the possible targets of miR-195. The results showed that the binding site of miR-195 was in the 3'-UTR of CARM1 (Figure 4A). Then dual-luciferase reporter assay was used to identify the relationship between CARM1 and miR-195. Results revealed that luciferase activity in HCT-116 and HT-29 cells co-transfected with WT-CARM1-PGL3 and miR-195 was strikingly decreased compared with controls, while cells co-transfected with MUT-CARM1-PGL3 and miR-195 showed no obvious changes in luciferase activity (Figure 4B and C). Moreover, miR-195 inhibition reduced the luciferase activity of WTCARM1-PGL3, but had no effect on the luciferase activity of MUT-CARM1-PGL3 (Figure 4D and E). To further confirm whether miR-195 can affect the expression of CARM1, mi-control, miR-195, or anti-miR-195 were transfected into cells to investigate the mRNA and protein levels of CARM1 using qRT-PCR and Western blot. Results showed that enforced expression of miR-195 significantly reduced the protein level of CARM1, whereas miR-195 downregulation increased the CARM1 expression in HCT-116 and HT-29 cells (Figure $4 \mathrm{G}$ and $\mathrm{H}$ ). However, it was found that there were no obvious differences in CARM1 mRNA level among miR-195, anti-miR-195, and miR-control groups (Figure 4F). Taken together, these results validated that miR-195 targetedly 


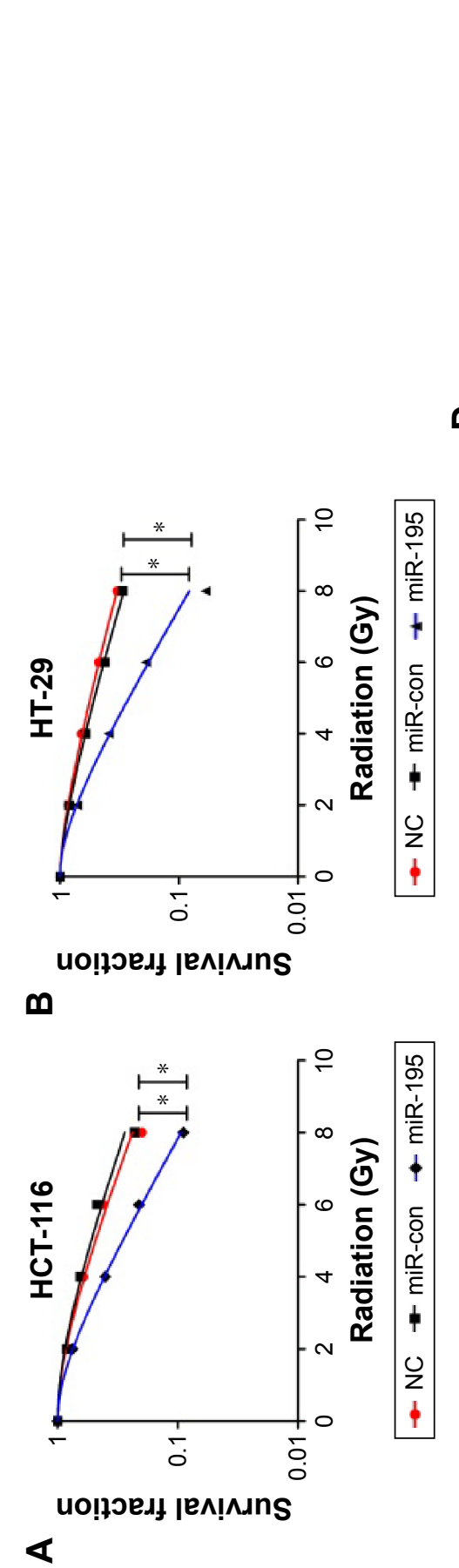

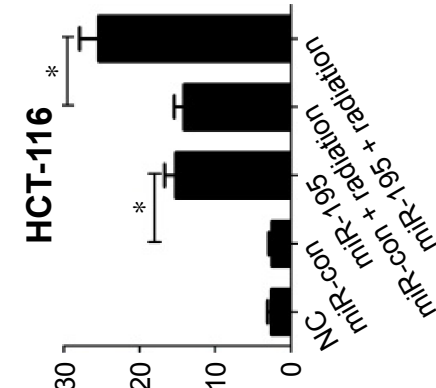

(\%) әреג

D s!soldod $\forall$

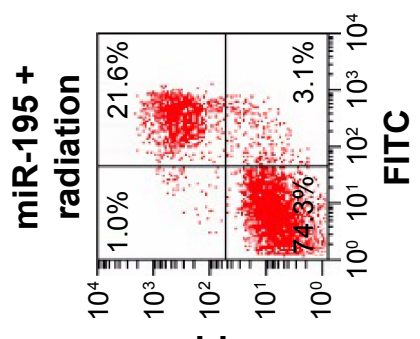

Id

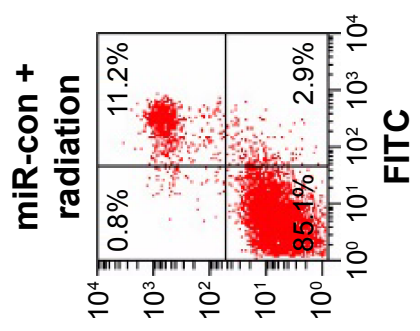

Id

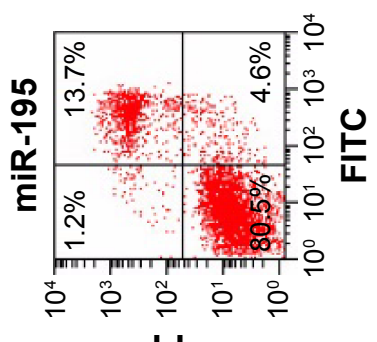

Id

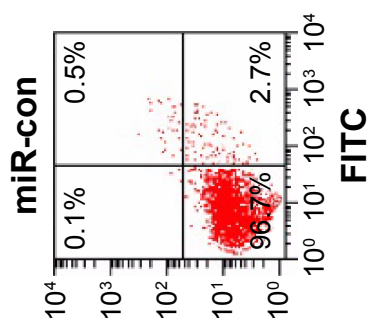

Id

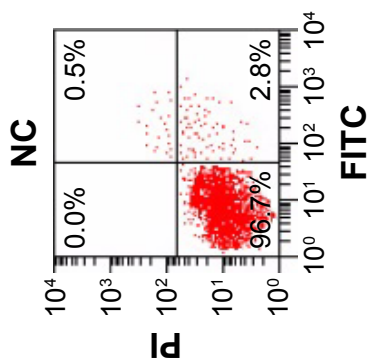

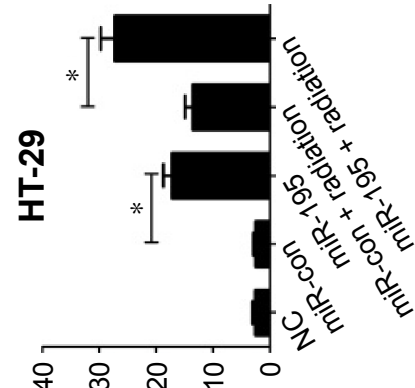

(\%) әㄹ.

ᄂ $\quad$ s!soldod $\forall$
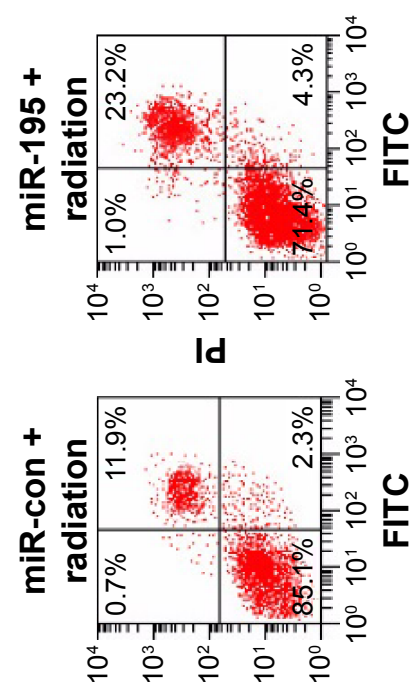

Id
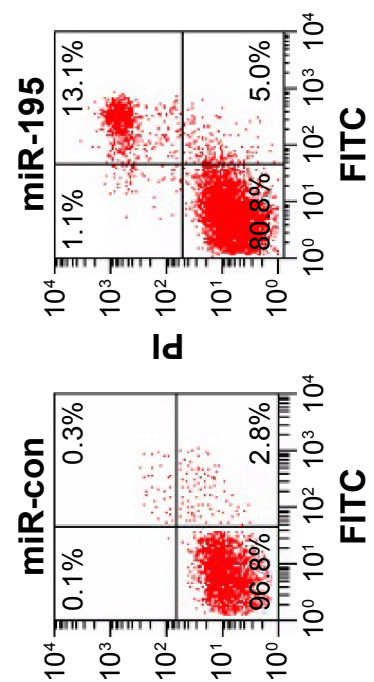

Id

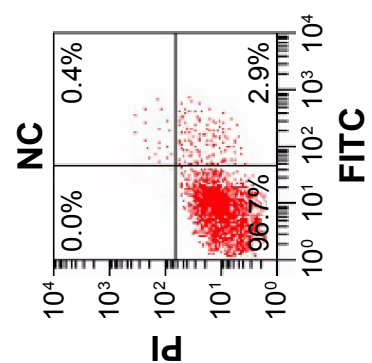

$6 Z-\perp H$ 


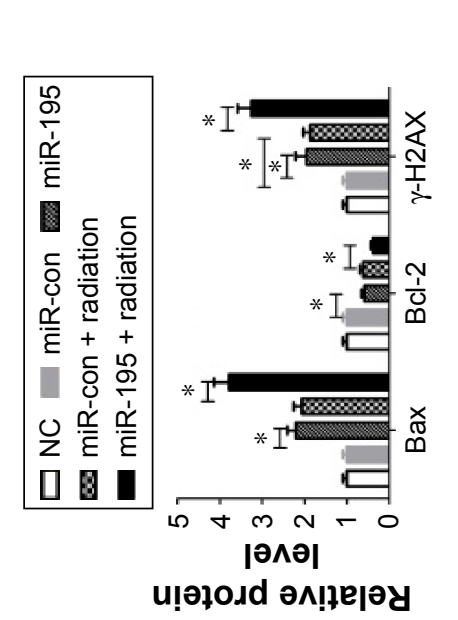

regulated CARM1 expression at the posttranscriptional level in HCT-116 and HT-29 cells.

\section{Restoration of CARMI expression relieves the enhanced radiosensitivity of CRC cells mediated by miR-195}

To further elucidate whether miR-195 enhanced radiosensitivity of CRC cells through regulating CARM1, HCT-116 and HT-29 cells were transfected with miR-control or miR-195 mimics or co-transfected miR-195 mimics with pcDNA-control or pcDNA-CARM1. Western blot analysis showed that pcDNA-CARM1 could rescue the reduced level of CARM1 by miR-195 overexpression in HCT-116 and HT-29 cells (Figure 5A), which suggested that the rescue assay was successful. Transfected cells were irradiated with $0,2,4,6$, and 8 Gy X-rays for clonogenic assay or a single dose of 4-Gy X-ray for apoptosis assay. The clonogenic assay and flow cytometry demonstrated that ectopic expression of CARM1 by pcDNA-CARM1 significantly relieved the reduced survival fractions of cellular colony and the increased apoptosis triggered by miR-195 in HCT-116 and HT-29 cells (Figure 5B and C). In addition, Western blot manifested that restoration of CARM1 significantly reversed the effects of miR-195 on Bax, Bcl-2, and $\gamma$-H2AX expression in HCT-116 (Figure 5D) and HT-29 cells (Figure 5E). These results indicated that miR-195 enhanced radiosensitivity of CRC cells by targeting CARM1.

\section{Effect of miR-I95 on radiosensitivity of xenografted tumor in vivo}

To investigate the role of miR-195 on radiosensitivity in a mouse xenotransplant model of colon tumor, HT-29 cells overexpressing miR-195 or miR-control were injected subcutaneously into nude mice. The mice were treated with a single dose of 8-Gy X-rays when the tumor volume was about $100 \mathrm{~mm}^{3}$. The body weight and tumor volume were measured every 5 days after radiation. As shown in Figure 6A, no significant difference was observed in the body weight among different groups (Figure 6A). Exposed to the same dose of radiation ( $8 \mathrm{~Gy}$ ), the weight (Figure 6C) and size (Figure 6B and D) of xenografts derived from miR-195overexpressed cells were dramatically decreased compared with those derived from miR-control treated cells. These results suggested that miR-195 increased the radiosensitivity of CRC cells in vivo.

\section{Discussion}

Radiotherapy is an important clinical treatment for patients with CRC. However, radioresistance is a challenging 
obstacle to this therapy. Therefore, more and more research studies should be conducted to deal with this issue. Radioresistance is involved in many complicated processes, including autophagy, ${ }^{22}$ angiogenesis, ${ }^{23}$ cancer stem cells, ${ }^{24}$ aberrant activation of signaling pathways, ${ }^{25}$ and abnormal expressions of DNA repair proteins. ${ }^{26}$ Several previous studies have reported that miRNAs could increase

A

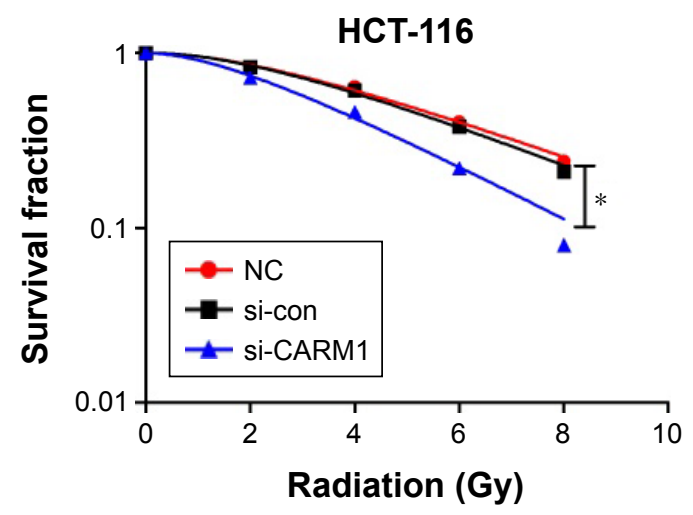

C

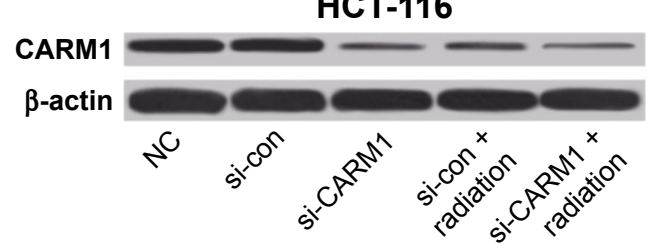

E

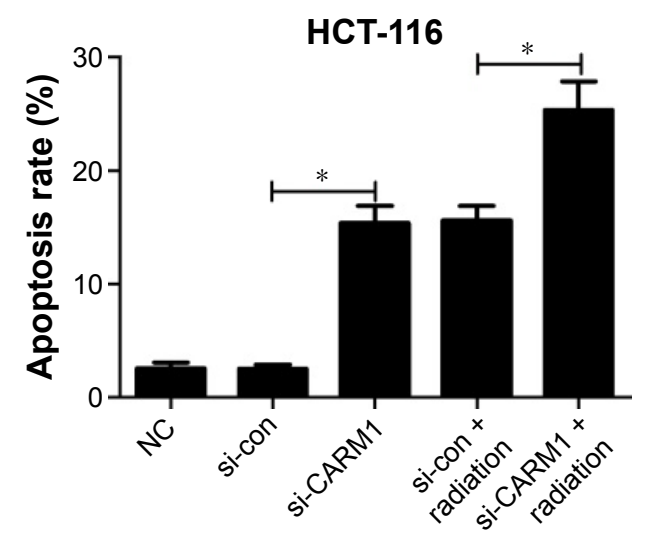

G

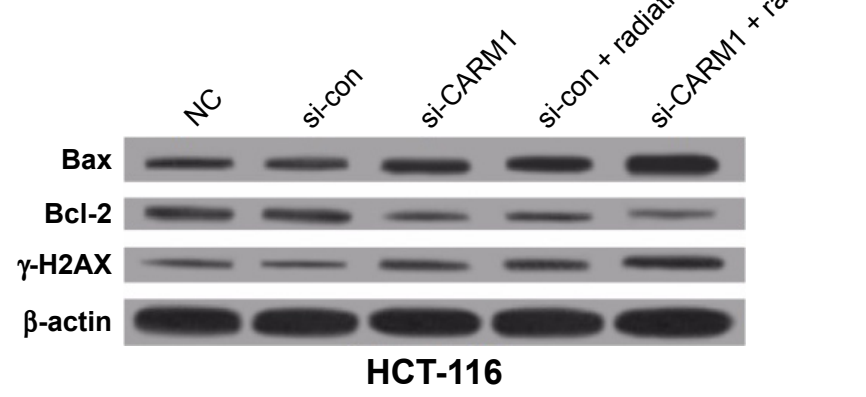

or decrease radiosensitivity of various tumors, ${ }^{27-29}$ which suggests that miRNAs may play vital roles in radiation resistance. Moreover, miRNAs could regulate radiation sensitivity and resistance of CRC. ${ }^{30-32}$ Zhang et al $^{32}$ found that miR-630 mediated by CREB could enhance radiosensitivity in CRC cell lines by inducing cell apoptosis and death through targeting BCL2L2 and TP53RK. miR-124 could

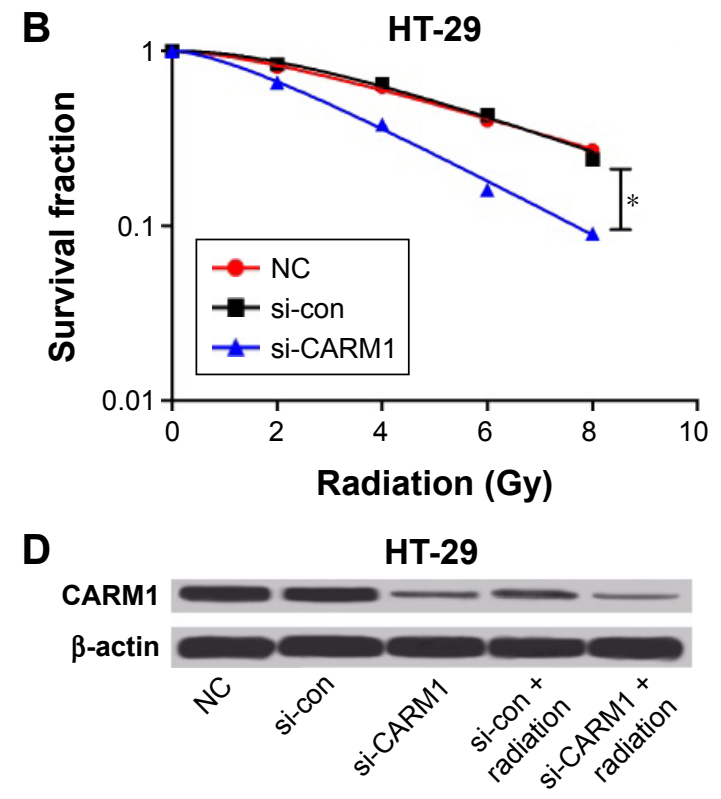

$\mathbf{F}$
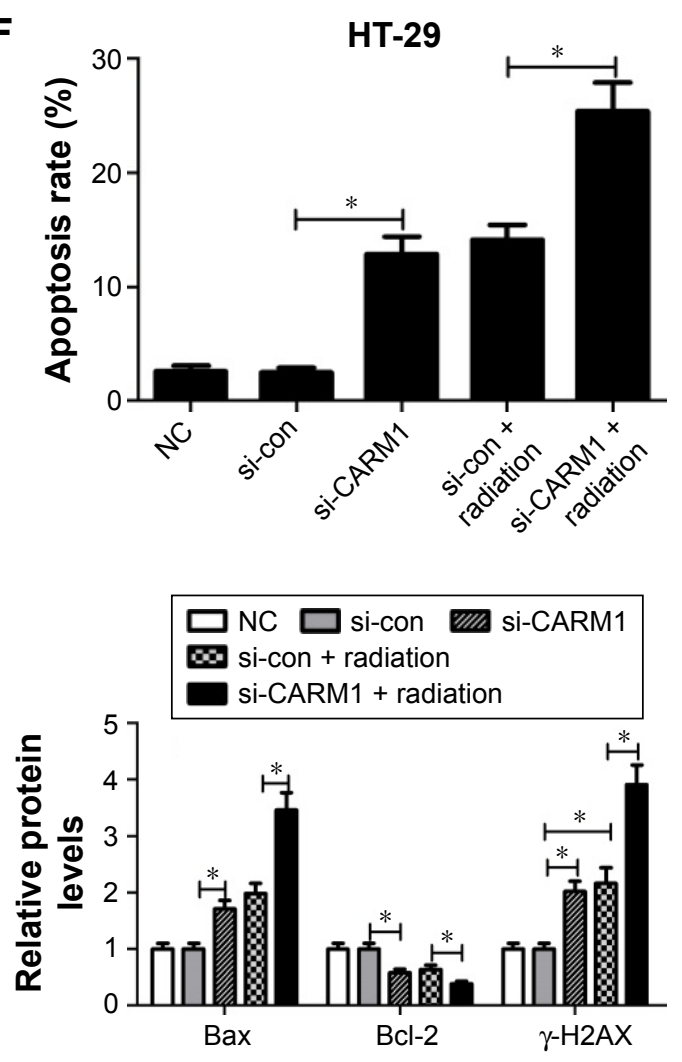

Figure 3 (Continued) 

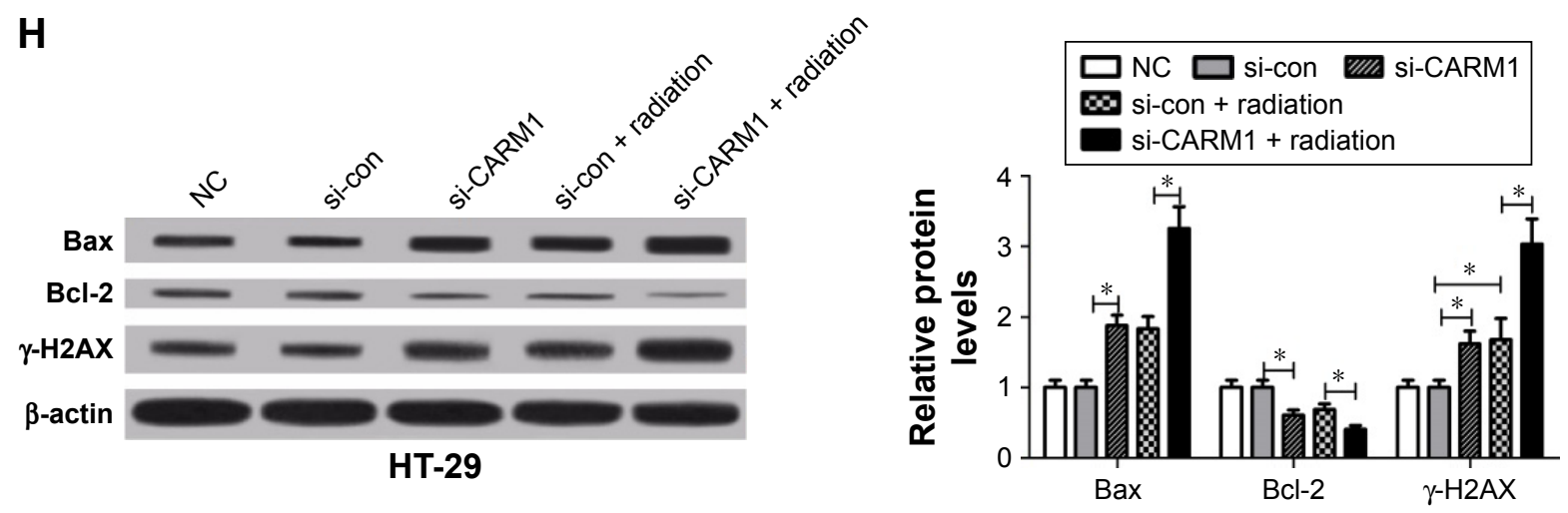

Figure 3 Effect of CARMI knockdown on the radiosensitivity of CRC cells.

Notes: HCT-II 6 and HT-29 cells were transfected with si-control (si-con) and si-CARMI at $24 \mathrm{~h}$ of incubation. Survival fractions were calculated in transfected HCT-II 6 (A) and HT-29 cells (B) after treatment with 0, 2, 4, 6, or 8 Gy of ionizing radiation for $24 \mathrm{~h}$. The CARMI level was detected using Western blot in HCT-I I6 (C) and HT-29 cells (D) after treatment with or without ionizing radiation (4 Gy) for $24 \mathrm{~h}$. Cell apoptosis was detected by flow cytometry in transfected HCT-I I6 (E) and HT-29 (F) cells after treatment with or without ionizing radiation ( $4 \mathrm{~Gy}$ ) for $24 \mathrm{~h}$. The levels of apoptosis-related proteins $\mathrm{Bax}, \mathrm{Bcl}-2$, and $\gamma$ - $\mathrm{H} 2 \mathrm{AX}$ were determined by Western blot in transfected HCT-II $6(\mathbf{G})$ and HT-29 cells $(\mathbf{H})$ after treatment with or without ionizing radiation (4 Gy) for 24 h. $* P<0.05$ vs si-control.

Abbreviations: NC, negative control; CRC, colorectal cancer.

increase the radiosensitivity of CRC cells by suppressing the expression of PRRX1, and further reverse epithelial mesenchymal transition (EMT) and simultaneously weaken the self-renewal properties of cells. ${ }^{30} \mathrm{miR}-100$ upregulation could radiosensitize CCL-244 cells in CRC by leading to apoptosis and DNA double-strand breaks. ${ }^{33}$ The improvements of miRNAs in CRC radiosensitivity were consistent with our findings. In the present study, we revealed that

A CARM1 3 '-UTR-WT 5'...ACAACGgacGUGACAUGCUGCUU... miR-195 3' CGGUUAUAAAGACACGACGAU

CARM1 3'-UTR-MUT 5 '...ACAACGGACGUGACACUGGUUCU...

B

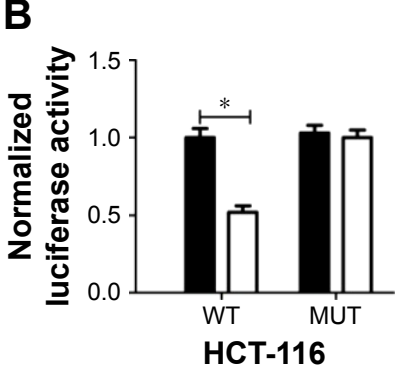

$\square$ miR-con $\square$ miR-195

$\mathbf{F}$
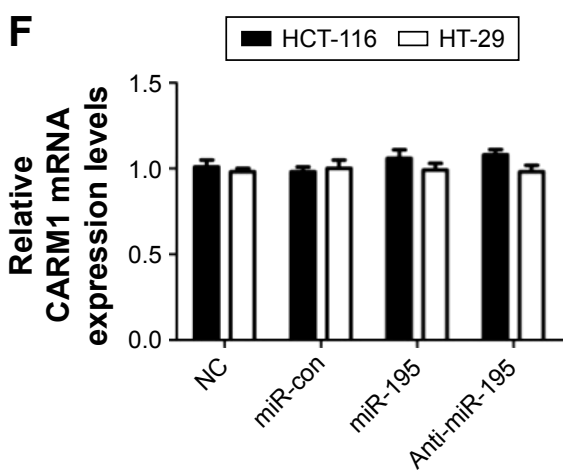

C

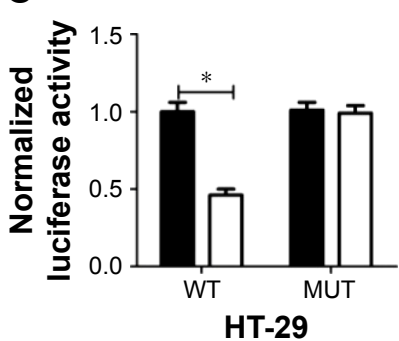

HT-29
D

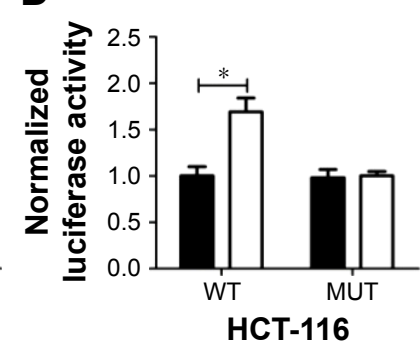

E

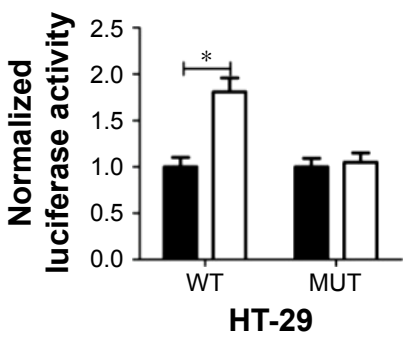

miR-con $\square$ Anti-miR-34a

G
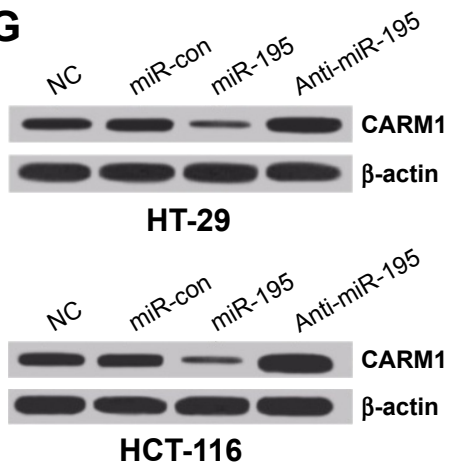

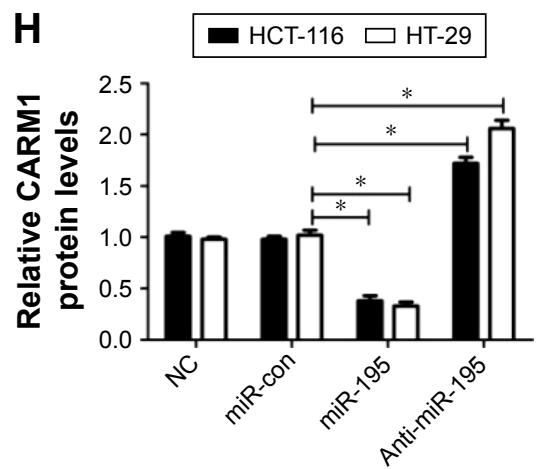

Figure 4 miR-195 directly targets 3'-UTR of CARMI and regulates its expression in CRC cells.

Notes: (A) Wild type (WT) and mutant (MUT) 3'-UTR binding sites for miR-195 are shown. The mutated bases are labeled with a horizontal line. (B and C) The relative luciferase activity was measured in HCT-II6 and HT-29 cells co-transfected WT or MUT CARMI 3'-UTR with miR-195 mimic or miR-control. (D and E) The relative luciferase activity was measured in HCT-II6 and HT-29 cells co-transfected WT or MUT CARMI 3'-UTR with anti-miR-I95 or anti-miR-control. The relative mRNA (F) and protein (G and $\mathbf{H})$ level of CARMI was detected in HCT-II6 and HT-29 cells transfected with miR-195 mimics or anti-miR-195. $* P<0.05$ vs miR-control.

Abbreviations: NC, negative control; CRC, colorectal cancer. 
expression of miR-195 was significantly downregulated in CRC cell lines HCT-116 and HT-29 compared with HCEpiC cells. Moreover, overexpression of miR-195 could enhance the radiosensitivity of CRC, evidenced by reduced survival fractions and induced apoptosis after miR-195 overexpression. Similarly, a previous study revealed that miR-195 enhanced the radiosensitivity of breast cancer cells by inhibiting the anti-apoptosis protein BCL-2. ${ }^{19}$ This study and our study both suggested that miR-195 could modify the outcome of radiation by the induction of apoptosis. Contrary
A

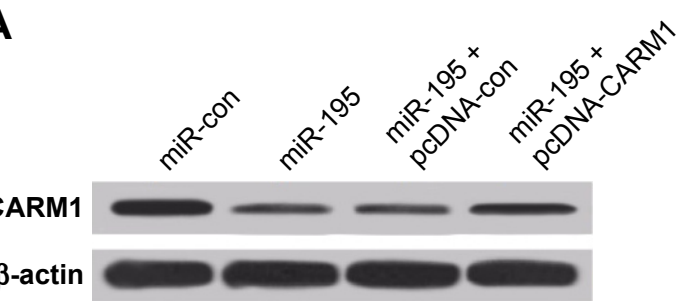

HCT-116

B

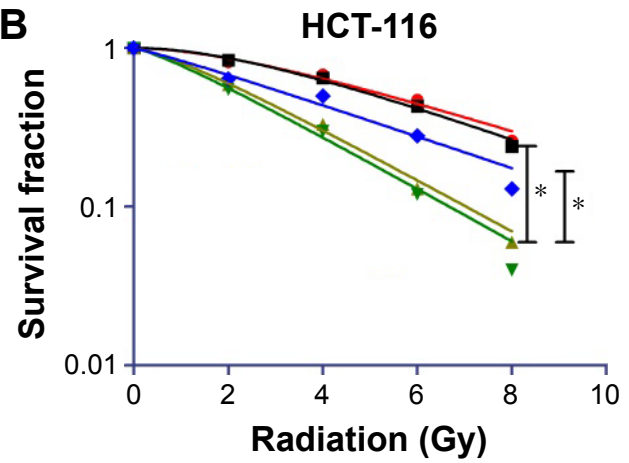

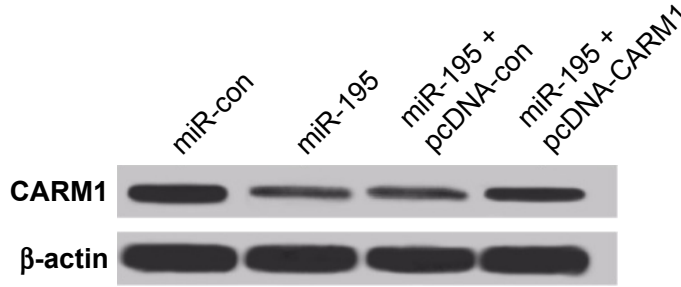

HT-29

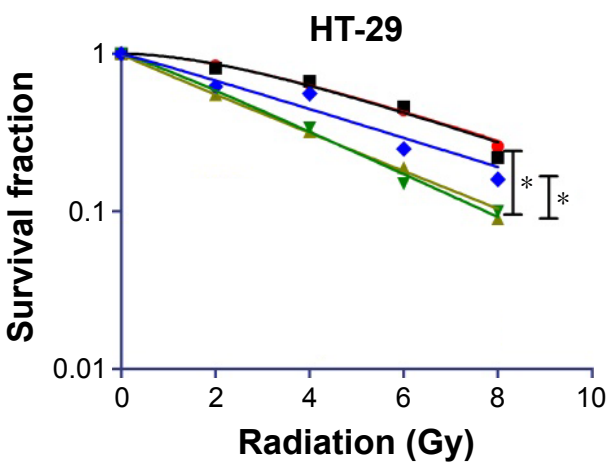

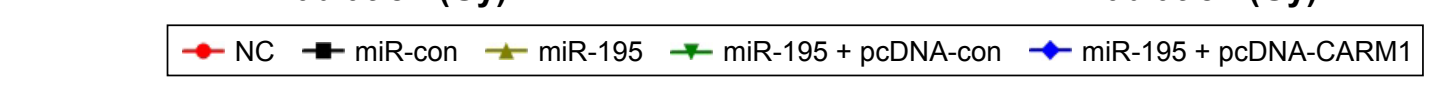

C

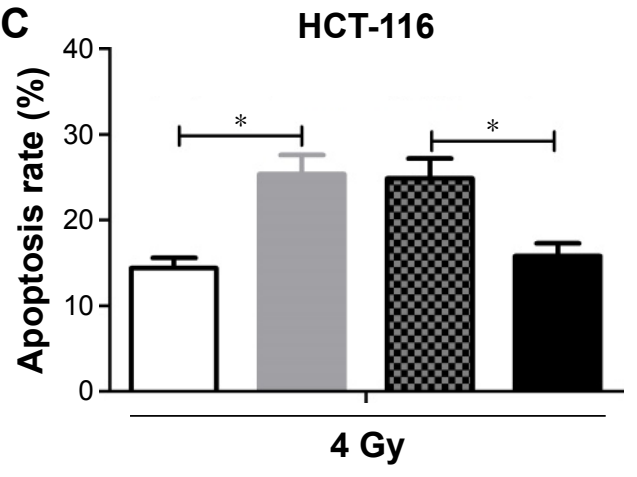

HT-29

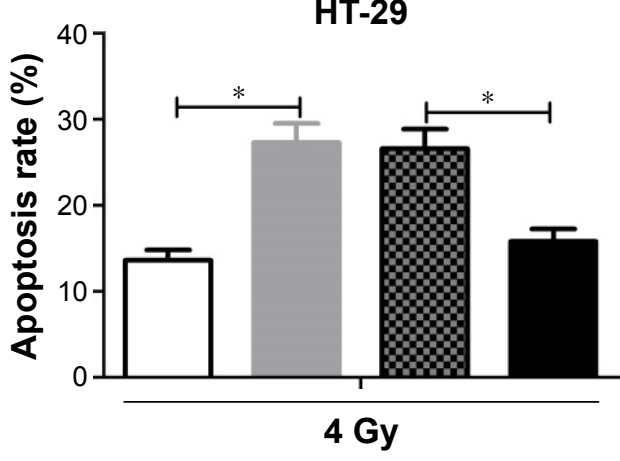

miR-195 + pcDNA-CARM1

D

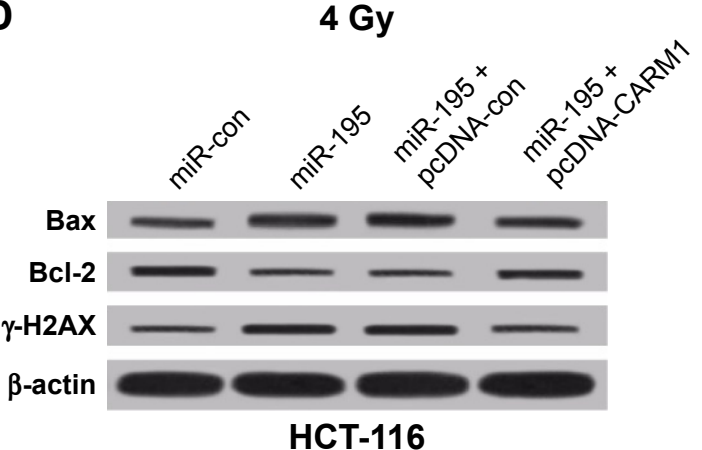

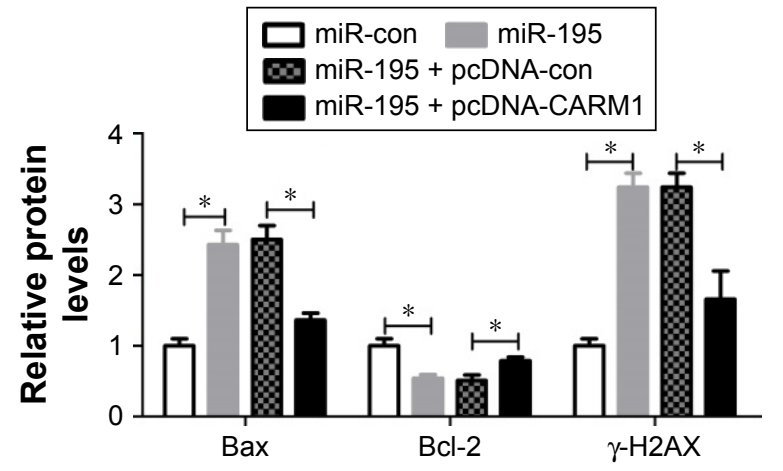

Figure 5 (Continued) 

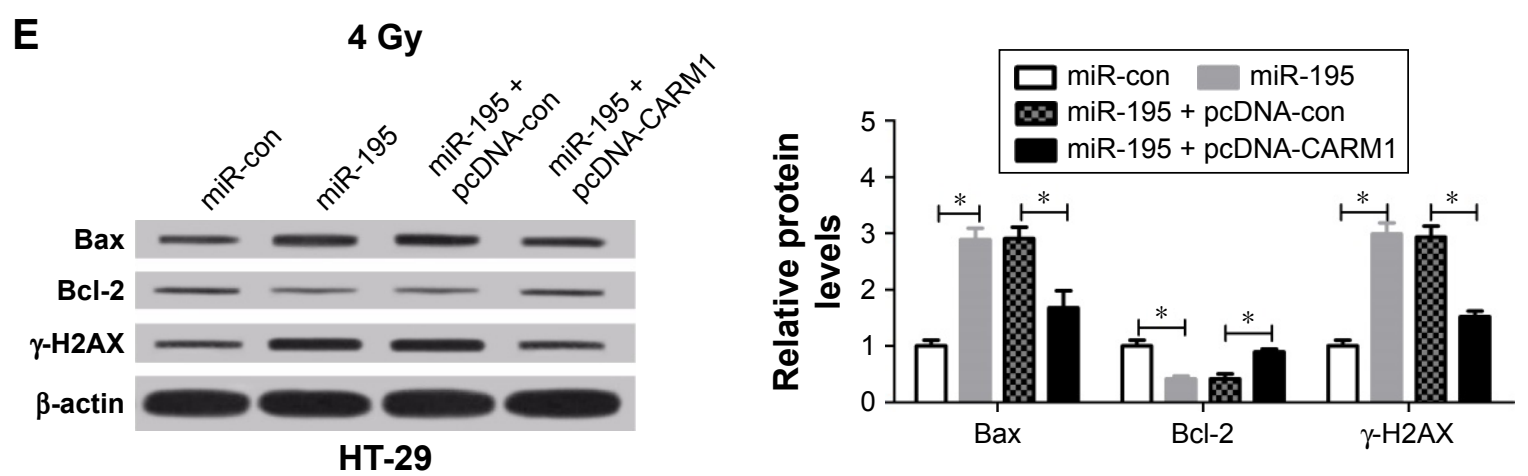

Figure 5 miR-195 enhances radiosensitivity of CRC cells by regulating CARMI.

Notes: HCT-II6 and HT-29 cells were transfected with miR-control or miR-195 mimics or co-transfected miR-195 mimics with pcDNA-control (pcDNA con) or pCDNA-CARMI. (A) The level of CARMI was detected by Western blot. (B) Survival fractions were estimated in transfected HCT-I I6 and HT-29 cells after treatment with $0,2,4,6$, or $8 \mathrm{~Gy}$ of ionizing radiation. (C) Cell apoptosis assay of flow cytometry in transfected HCT-II 6 and HT-29 cells after treatment with ionizing radiation (4 Gy). Apoptosis-related proteins Bax, Bcl-2, and $\gamma$-H2AX were detected by Western blot in transfected HCT-II 6 (D) and HT-29 (E) cells after treatment with ionizing radiation (4 Gy). ${ }^{*} P<0.05$ vs control groups.

Abbreviations: NC, negative control; CRC, colorectal cancer.

to our results, Xue et al ${ }^{34}$ found that miR-221 could reduce the radiosensitivity of CRC cells by regulating PTEN, negatively regulating the AKT/PKB signaling pathway. In addition, miR-106b could induce cell radioresistance in CRC by directly targeting PTEN and p21, accompanied by tumor-initiating cell capacity enhancement. ${ }^{31}$

A

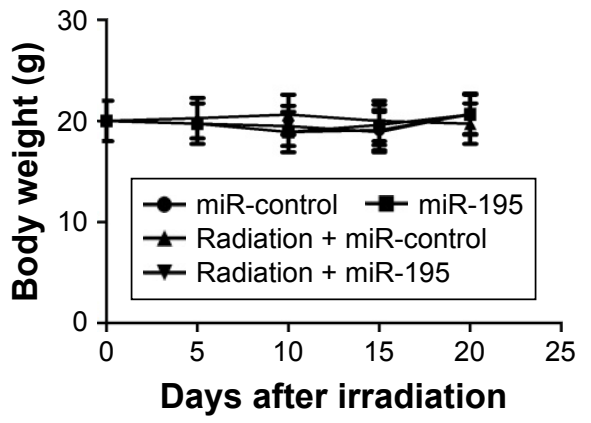

C

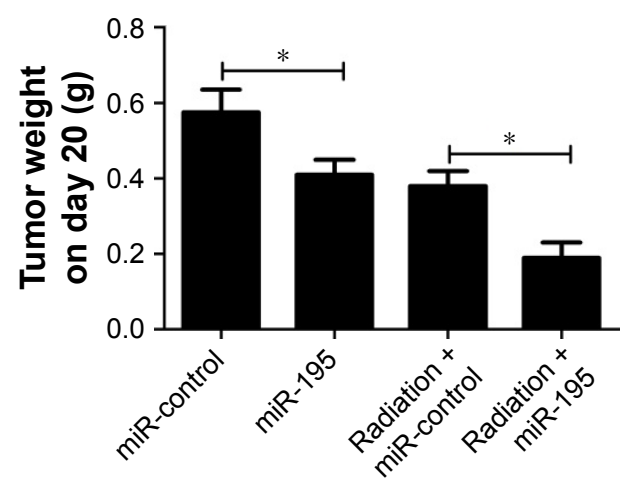

A previous study indicated that CARM1 played pleiotropic roles in cell proliferation and survival in breast cancer. ${ }^{35}$ A study also showed that CARM1 was highly overexpressed in CRC. ${ }^{36}$ However, the role of CARM1 in radiosensitivity of cancers is unknown. In the present study, we found that knockdown of CARM1 inhibited colony
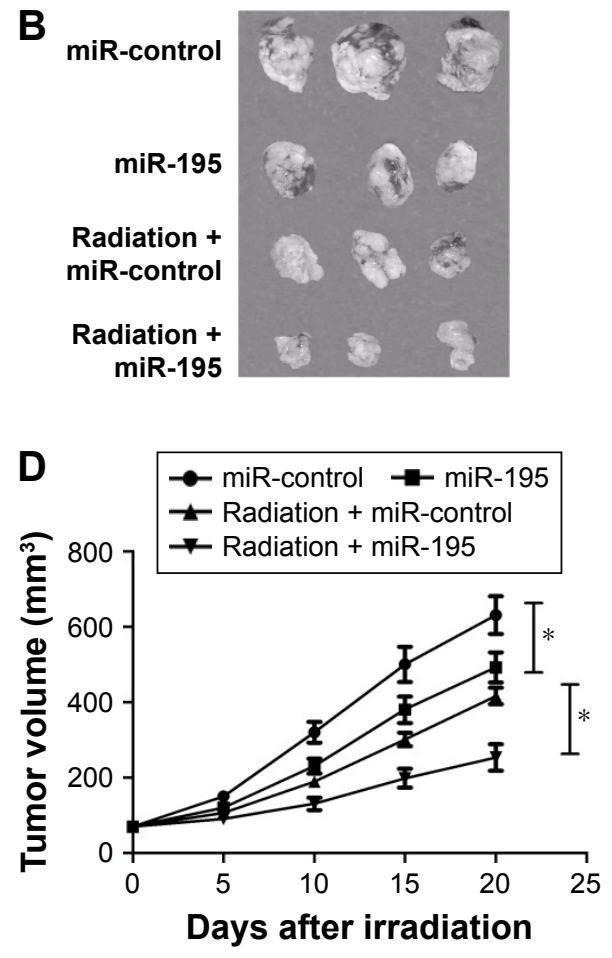

Figure 6 miR-195 upregulation increases CRC cell radiosensitivity in vivo.

Notes: HT-29 cells overexpressing miR-195 or miR-control were subcutaneously injected into nude mice aged 4-6 weeks. Radiation was performed with 8 Gy X-rays when the tumor volume was about $100 \mathrm{~mm}^{3}$. (A) Body weight of the mouse was determined every 5 days after radiation in different groups. (B) Image of representative tumor in different groups at Day 20 after radiation. (C) Weight of tumor in different groups at Day 20 after radiation. (D) Tumor growth curve in different groups. $* P<0.05$ vs control groups.

Abbreviation: CRC, colorectal cancer. 
survival and induced apoptosis. A previous study indicated that CARM1 exerted a biological effect in colon cancer cells by regulating $\mathrm{p} 53$ and NF- $\mathrm{\kappa B}$ target gene expression, ${ }^{36}$ which were involved in the radiosensitivity of CRC cells. ${ }^{37,38}$ These studies suggested that CARM1 may regulate the radiosensitivity of CRC cells through p53 and NF- $\mathrm{BB}$; this needs further studies to confirm. Furthermore, a dual-luciferase assay was performed and CARM1 was verified to be a target of miR-195. The expression of CARM1 at protein levels was regulated by miR-195, and the restoration of CARM1 expression reversed the enhanced radiosensitivity of CRC cells induced by miR-195. Moreover, a previous study found a regulatory loop between CARM1 and miR-223 that controls the differentiation of $\mathrm{CD}^{34+}$ toward the myeloid lineage. ${ }^{39}$ Our findings suggest that miR-195 enhances the radiosensitivity of CRC cells by inhibiting the CARM1 expression, providing a theoretical foundation for the clinical application of miRNAs in therapy of radioresistance.

\section{Conclusion}

In summary, the expression of miR-195 was downregulated in CRC cell lines HCT-116 and HT-29. Enforced expression of miR-195 increased the radiosensitivity of CRC cells, at least partially, by inhibiting CARM1 expression. Our findings suggest that the restoration of miR-195 could be a promising therapeutic strategy for the radioresistance of CRC.

\section{Disclosure}

This research did not receive any specific grant from funding agencies in the public, commercial, or not-for-profit sectors. The authors report no conflicts of interest in this work.

\section{References}

1. Puente Gutiérrez J, Moreno M, Domínguez Jiménez J, Bernal Blanco E, Díaz Iglesias J. Effectiveness of a colonoscopic screening programme in first-degree relatives of patients with colorectal cancer. Colorectal Dis. 2011;13(6):e145-e153.

2. Rundle AG, Lebwohl B, Vogel R, Levine S, Neugut AI. Colonoscopic screening in average-risk individuals ages 40 to 49 vs 50 to 59 years. Gastroenterology. 2008;134(5):1311-1315.

3. Ahmed M, Al-Attar A, Kim J, et al. CD24 shows early upregulation and nuclear expression but is not a prognostic marker in colorectal cancer. J Clin Pathol. 2009;62(12):1117-1122.

4. Covic M, Hassa PO, Saccani S, et al. Arginine methyltransferase CARM1 is a promoter-specific regulator of NF- $\mathrm{KB}$-dependent gene expression. EMBO J. 2005;24(1):85-96.

5. An W, Kim J, Roeder RG. Ordered cooperative functions of PRMT1, p300, and CARM1 in transcriptional activation by p53. Cell. 2004;117(6): $735-748$.

6. Wang L, Zhao Z, Meyer MB, et al. CARM1 methylates chromatin remodeling factor BAF155 to enhance tumor progression and metastasis. Cancer Cell. 2014;25(1):21-36.

7. Wang L, Zeng H, Wang Q, et al. MED12 methylation by CARM1 sensitizes human breast cancer cells to chemotherapy drugs. Sci Adv. 2015;1(9): e1500463.
8. Bartel DP. MicroRNAs: genomics, biogenesis, mechanism, and function. Cell. 2004;116(2):281-297.

9. Ambros V. The functions of animal microRNAs. Nature. 2004; 431(7006):350-355.

10. Garzon R, Calin GA, Croce CM. MicroRNAs in cancer. Аnпи Rev Med. 2009;60(1):167-179.

11. Metheetrairut $\mathrm{C}$, Slack FJ. MicroRNAs in the ionizing radiation response and in radiotherapy. Curr Opin Genet Dev. 2013;23(1):12-19.

12. Xiao $\mathrm{S}$, Yang $Z$, Lv R, et al. miR-135b contributes to the radioresistance by targeting GSK $3 \beta$ in human glioblastoma multiforme cells. PloS One. 2014;9(9):e108810.

13. Sun Q, Liu T, Yuan Y, et al. MiR-200c inhibits autophagy and enhances radiosensitivity in breast cancer cells by targeting UBQLN1. Int J Cancer. 2015;136(5):1003-1012.

14. Duan W, Xu Y, Dong Y, et al. Ectopic expression of miR-34a enhances radiosensitivity of non-small cell lung cancer cells, partly by suppressing the LyGDI signaling pathway. J Radiat Res. 2013;54(4): 611-619.

15. Lin J, Liu C, Gao F, et al. miR-200c enhances radiosensitivity of human breast cancer cells. J Cell Biochem. 2013;114(3):606-615.

16. Li D, Zhao Y, Liu C, et al. Analysis of MiR-195 and MiR-497 expression, regulation and role in breast cancer. Clin Cancer Res. 2011;17(7): $1722-1730$.

17. Wang X, Wang J, Ma H, Zhang J, Zhou X. Downregulation of miR-195 correlates with lymph node metastasis and poor prognosis in colorectal cancer. Med Oncol. 2012;29(2):919-927.

18. Liu L, Chen L, Xu Y, Li R, Du X. microRNA-195 promotes apoptosis and suppresses tumorigenicity of human colorectal cancer cells. Biochem Biophys Res Commun. 2010;400(2):236-240.

19. Zhu J, Ye Q, Chang L, et al. Upregulation of miR-195 enhances the radiosensitivity of breast cancer cells through the inhibition of BCL-2. Int J Clin Exp Med. 2015;8(6):9142-9148.

20. Hung PS, Kao SY, Shih YH, et al. Insulin-like growth factor binding protein-5 (IGFBP-5) suppresses the tumourigenesis of head and neck squamous cell carcinoma. J Pathol. 2008;214(3):368-376.

21. Yaromina A, Krause M, Thames H, et al. Pre-treatment number of clonogenic cells and their radiosensitivity are major determinants of local tumour control after fractionated irradiation. Radiother Oncol. 2007; 83(3):304-310.

22. Chaachouay H, Ohneseit P, Toulany M, et al. Autophagy contributes to resistance of tumor cells to ionizing radiation. Radiother Oncol. 2011; 99(3):287-292.

23. Francescone RA, Scully S, Faibish M, et al. Role of YKL-40 in the angiogenesis, radioresistance, and progression of glioblastoma. J Biol Chem. 2011;286(17):15332-15343.

24. Moncharmont C, Levy A, Gilormini M, et al. Targeting a cornerstone of radiation resistance: cancer stem cell. Cancer Lett. 2012;322(2): 139-147.

25. Dumont FJ, Bischoff P. Disrupting the mTOR signaling network as a potential strategy for the enhancement of cancer radiotherapy. Curr Cancer Drug Targets. 2012;12(8):899-924.

26. Beskow C, Skikuniene J, Holgersson A, et al. Radioresistant cervical cancer shows upregulation of the NHEJ proteins DNA-PKcs, Ku70 and Ku86. Br J Cancer. 2009;101(5):816-821.

27. Deng X, Ma L, Wu M, et al. miR-124 radiosensitizes human glioma cells by targeting CDK4. J Neurooncol. 2013;114(3):263-274.

28. Wang P, Zhang J, Zhang L, et al. MicroRNA 23b regulates autophagy associated with radioresistance of pancreatic cancer cells. Gastroenterology. 2013;145(5):1133-1143.

29. Liu YJ, Lin YF, Chen YF, et al. MicroRNA-449a enhances radiosensitivity in CL1-0 lung adenocarcinoma cells. PLoS One. 2013;8(4): e62383.

30. Zhang Y, Zheng L, Huang J, et al. MiR-124 Radiosensitizes human colorectal cancer cells by targeting PRRX1. PLoS One. 2014;9(4): e93917.

31. Zheng L, Zhang Y, Liu Y, et al. MiR-106b induces cell radioresistance via the PTEN/PI3K/AKT pathways and $\mathrm{p} 21$ in colorectal cancer. $J$ Transl Med. 2015;13(1):252. 
32. Zhang Y, Yu J, Liu H, et al. Novel Epigenetic CREB-miR-630 Signaling axis regulates radiosensitivity in colorectal cancer. PloS One. 2015; 10(8):e0133870.

33. Yang XD, Xu XH, Zhang SY, et al. Role of miR-100 in the radioresistance of colorectal cancer cells. Am J Cancer Res. 2015;5(2):545-559.

34. Xue Q, Sun K, Deng HJ, et al. Anti-miRNA-221 sensitizes human colorectal carcinoma cells to radiation by upregulating PTEN. World J Gastroenterol. 2013;19(48):9307-9317.

35. Al-Dhaheri M, Wu J, Skliris GP, et al. CARM1 is an important determinant of ER $\alpha$-dependent breast cancer cell differentiation and proliferation in breast cancer cells. Cancer Res. 2011;71(6):2118-2128.
36. Kim YR, Lee BK, Park RY, et al. Differential CARM1 expression in prostate and colorectal cancers. BMC Cancer. 2010;10(1):197.

37. Zhang Y, Wei Y, Zhu Z, et al. Icariin enhances radiosensitivity of colorectal cancer cells by suppressing NF- $\mathrm{BB}$ activity. Cell Biochem Biophys. 2014;69(2):303-310.

38. Chiu SJ, Hsaio $\mathrm{CH}$, Tseng $\mathrm{HH}$, et al. Rosiglitazone enhances the radiosensitivity of p53-mutant HT-29 human colorectal cancer cells. Biochem Biophys Res Commun. 2010;394(3):774-779.

39. Vu LP, Zhao X, Perna F, et al. Downregulation of protein arginine methyltransferase-4 (PRMT4) promotes MiR-223 expression and myeloid differentiation. Blood. 2010;116(21):3632.

\section{Publish your work in this journal}

OncoTargets and Therapy is an international, peer-reviewed, open access journal focusing on the pathological basis of all cancers, potential targets for therapy and treatment protocols employed to improve the management of cancer patients. The journal also focuses on the impact of management programs and new therapeutic agents and protocols on

\section{Dovepress}

patient perspectives such as quality of life, adherence and satisfaction The manuscript management system is completely online and includes a very quick and fair peer-review system, which is all easy to use. Visit http://www.dovepress.com/testimonials.php to read real quotes from published authors. 